\section{A possible interaction between lithium and rofecoxib}

A 73 year old man who has suffered from manic depressive illness for 40 years had continuous prophylactic treatment with lithium citrate since 1969. Over the past 6 years, the antidepressant lofepramine in daily doses between 70 and $140 \mathrm{mg}$ had also been used due to episodic mild depression during the winter months. In 1994, he successfully underwent coronary bypass surgery and aortic valve replacement. Following surgery, chronic warfarin therapy was started. In addition, over the past 2 years his cardiovascular drug regimen was frusemide $40 \mathrm{mg}$ daily, captopril $6.25 \mathrm{mg}$ twice daily and sotalol $80 \mathrm{mg}$ twice daily. Laboratory tests on a yearly basis for the past 13 years had shown values within age-corrected reference values for serum creatinine $\left(100-120 \mu \mathrm{mol}^{-1}\right)$ and Cr-EDTA clearance $\left(61-82 \mathrm{ml} \mathrm{min}^{-1}\right)$. The $12 \mathrm{~h}$ serum lithium concentrations ranged between 0.6 and $0.9 \mathrm{mmol} 1^{-1}$ over the same time period. However, lithium doses had been reduced over the past decade from $12 \mathrm{mmol}\left(84 \mathrm{mg} \mathrm{Li}^{+}\right)$twice daily to $6 \mathrm{mmol}\left(42 \mathrm{mg} \mathrm{Li}^{+}\right)$ twice daily in order to keep the serum lithium concentrations within laboratory guidelines and to maintain a sustained theraputic effect.

In November 2000, the patient developed daytime somnolence and mild confusion, which was acute in onset. He was increasingly irritable and suffered from tremor as well as gait disturbances. A drug history revealed that he had been started on the cyclooxygenase-2 (COX-2) selective inhibitor rofecoxib $12.5 \mathrm{mg}$ daily 9 days earlier due to pain from trochanteritis of the left hip. The patient was not taking any other medicines including over-thecounter drugs or herbal medicines. Roxfecoxib was chosen in order to avoid a possible drug interaction with warfarin. The pain from the left hip disappeared gradually within a few days; however, the patient in parallel also developed clinical signs of lithium intoxication. The acute $12 \mathrm{~h}$ serum lithium concentration was $1.50 \mathrm{mmol} \mathrm{l}^{-1}$ and the serum creatinine was $143 \mu \mathrm{mol} 1^{-1}$. Lithium and rofecoxib were withdrawn (all other drugs were continued) and the clinical signs resolved within 1 week. The serum lithium concentration returned to levels observed before the start of rofecoxib treatment and the lithium therapy could was restarted. However, the lithium maintenance dose was reduced to $6 \mathrm{mmol}\left(42 \mathrm{mg} \mathrm{\textrm {Li } ^ { + }}\right)$ daily with a proportional $50 \%$ decrease in the observed maintenance serum lithium concentrations. The serum lithium and creatinine concentrations prior to, during, and after this episode are shown in Figure 1.

Litium nephrotoxicity is well recognized and characterized by progressive combined glomerular and tubulo-interstitinal nephropathy [1]. NSAIDs that are nonselective inhibitors of COX are known to decrease renal glomerular filtration rate and increase lithium serum concentration when used concomitantly [2]. Less information is available on the potential nephrotoxicity of the recently introduced selective COX-2 inhibiting NSAIDs, although effects on renal function similar to those of nonspecific NSAIDs have been reported [3]. Moreover, case reports of reversible acute renal failure in patients with chronic renal insufficiency treated with COX-2 inhibitors have been published [4]. In line with the observations reported here, there has also recently been another report of increased serum lithium concentrations and clinical signs of mild lithium toxicity following concomitant use of lithium and rofecoxib [5].

In summary, the patient developed clinical signs of mild to moderate lithium intoxication, which was confirmed by laboratory data of increased serum lithium and creatinine concentrations. These observations occurred with the combined use of lithium and rofecoxib, and following dechallenge of both drugs, the clinical status and laboratory data returned to expected levels. Both these drugs have been associated with nephrotoxicity and it is consequently likely that the lithium intoxication was induced by concomitant administration of rofecoxib causing a reversible decrease in renal function. However,

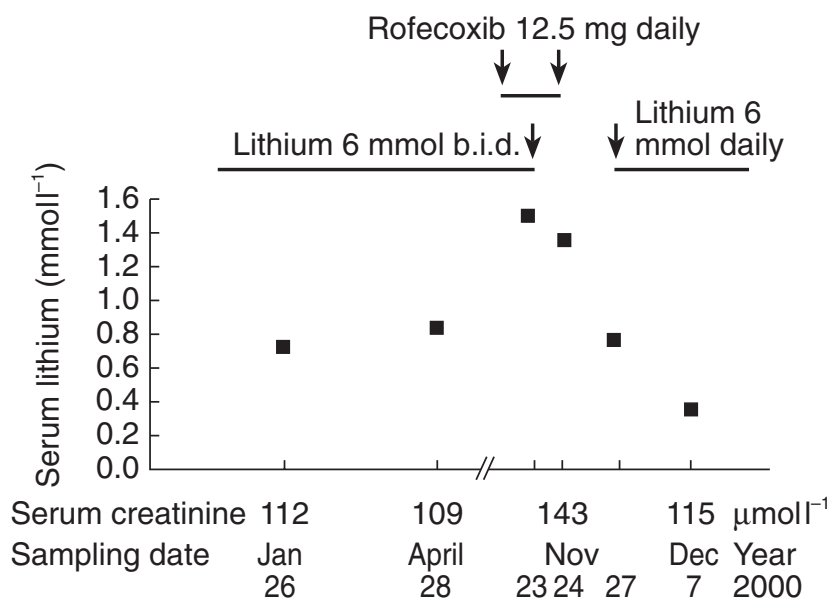

Figure 1 Serum lithium and creatinine concentrations before, during, and after lithium and rofecoxib co-administration. 
a more confirmatory re-challenge could not be performed for ethical reasons, and thus further observations are warranted for confirmation of the causal nature of the reported interaction.

\section{Jöns Lundmark, Tove Gunnarsson \& Finn Bengtsson \\ Department of Neuroscience and Locomotion, Division of Psychiatry. University Hospital, SE-581 85 Linköping, Sweden}

Received 12 March 2001, accepted 5 December 2001

Correspondence: Dr Jöns Lundmark, Department of Neuroscience and Locomotion, Division of Psychiatry. University Hospital, SE-581 85 Linköping, Sweden. E-mail: jons.lundmark@lio.se

\section{References}

1 Markowitz G, Radhakrishnan J, Kambham N, Valeri A, Hines W, D’Agati V. Lithium nephrotoxicity. A combined glomerular and tubulointerstitial nephrotoxicity. $J$ Am Soc Nephrol 2000; 11: 1439-1448.

2 Finley PR, Warner MD, Peabody CA. Clinical relevance of drug interactions with lithium. Clin Pharmacokinet 1995; 29: 172-191.

3 Braiter C. Effects of nonsteroidal anti-inflammatory drugs on renal function: Focus on cyclooxygenase-2-selective inhibition. Am J Med 1999; 107: 65S-71S.

4 Perazella M, Eras J. Are COX-2 selective inhibitors nephrotoxic? Am J Kidney Dis 2000; 35: 937-940.

5 Sajbel TA, Carter GW, Wiley RB, et al. Pharmacokinetic effects of rofecoxib therapy on lithium. Pharmacotherapy 2001; 21: 308.

\section{Syncope following oral chloroquine administration in a hypertensive patient controlled on amlodipine}

Amlodipine has achieved global use and acceptability in the monotherapy of moderate to severe essential hypertension. Chloroquine is still the choice antimalarial drug in areas where there is little antimalarial resistance and for the treatment of rheumatological disorders (systemic lupus and rheumatoid arthritis) which may be associated with hypertension. Thus, there exists a high probability of the concurrent use of chloroquine with amlodipine.

We provide the first report to our knowledge of a possible severe adverse drug interaction between amlodipine and chloroquine in a hypertensive patient. A 48 year old male physician, who is a known hypertensive (initial blood pressure 172/110 $\mathrm{mmHg}$ ) had achieved optimal blood pressure control of 136/86 $\mathrm{mmHg}$ after oral amlodipine $5 \mathrm{mg}$ daily for 3 months, according to his medical records. He developed a slight frontal headache and fever, and self administered four tablets of chloroquine sulphate (total $600 \mathrm{mg}$ base) at about $09.00 \mathrm{~h}$, based on the presumptive self diagnosis of malaria fever. Two hours later, whilst he conducted a medical clinic, he became nauseated, dizzy and collapsed from the upright posture. The nurse on duty immediately called the medical team on the emergency roster. Upon arrival, the patient was conscious, alert, but disoriented. His extremities were cool and he was placed in a head-down position on a couch. The body temperature was $38.8^{\circ} \mathrm{C}$, the radial pulse was regular, of poor volume, and there was a bradycardia of 52 beats $\min ^{-1}$. The initial blood pressure was systolic $80 \mathrm{mmHg}$ and the diastolic was unrecordable by mercury sphygmomanometer or Omron-automatic sphygmomanometer.

An intravenous access was obtained for the infusion of $5 \%$ dextrose-saline $(0.9 \%)$ solution. Blood samples were obtained for blood sugar, chemistry and malaria parasites. After $1 \mathrm{~h}$ of infusion of the dextrose-saline (about $800 \mathrm{ml}$ ), the blood pressure rose to $110 / 75 \mathrm{mmHg}$, and the pulse to 84 beats $\min ^{-1}$, regular and good volume. 12 lead -ECG was unremarkable, there were no malaria parasites detected and the patient was ambulant $2 \mathrm{~h}$ after the onset of his syncope. His long-term recovery was also unremarkable and sustained.

Chloroquine alone causes a hypotensive response $[1,2]$ and increases forearm blood flow, by decreasing forearm vascular resistance [2]. Recent evidence indicates that chloroquine causes venodilation via the release of nitric oxide in human forearm veins [3]. Thus, chloroquine may reduce both preload and afterload, but this is rarely symptomatic in healthy adults after oral dosing. The oral administration of chloroquine in the presence of amlodipine in this hypertensive patient, appears to have triggered a vasovagal syncope, which was correctable by dextrose-saline infusion. Amlodipine releases nitric oxide from canine coronary microvessels [4] and lacidipine increases endothelial nitric oxide formation in human endothelium in hypertensive patients [5].

Stimulation of endothelial nitric oxide synthase may thus be a property common to long acting calcium antagonists. Chloroquine also blocks the fast inward $\mathrm{K}^{+}$ channels in feline myocytes [6]. The patient had no malaria parasitaemia, and hence mechanistically the syncope may be as a result of acute synergistic hypotensive, venodilator and cardiac effects of the amlodipine and chloroquine, possibly acting via augmented nitric oxide production and calcium channel blockade. Since malaria fever per se is associated with orthostatic intolerance, this possible adverse interaction may be unrecognized and unreported. We urge caution in patients receiving combined treatment with 1,4 dihydropyridine calcium antagonists and aminoquinoline antimalarial drugs. 


\section{A. A. Leslie Ajayi' \& A. Q. Adigun ${ }^{2}$}

${ }^{1}$ Department of Medicine, Baylor College of Medicine, Center of Cardiovascular Diseases, Texas, Southern University, 3100 Cleburne Avenue, Houston, TX 77004 and ${ }^{2}$ Department of Medicine, Division of Clinical Pharmacology, Indiana University School of Medicine, Indianopolis, IND, USA

Received 9 October 2001, accepted 14 December 2001

Correspondence: Professor A. A. L. Ajayi, Department of Medicine, Baylor College of Medicine, Center of Cardiovascular Diseases, Texas, Southern University, 3100 Cleburne Avenue, Houston, TX 77004, USA. Tel.: 713-313-4251; Fax: 713-313-4219; E-mail: adeajayi@aol.com

\section{References}

1 Looareesuwan S, White NJ, Chanthavanich P, et al. Cardiovascular toxicity and distribution kinetics of intravenous chloroquine. $\mathrm{Br} \mathrm{J} \mathrm{Clin}$ Pharmacol 1986; 22: 31-36.

2 Anigbogun CN, Adigun SA, Inyang I, Adegunloye BJ. Chloroquine reduces blood pressure and forearm vascular resistance and increases forearm flow in healthy young adults. Clin Physiol 1993; 13: 209-216.

3 Abiose AK, Grossmann M, Tangphao O, Hoffman BB, Blaschke TF. Chloroquine induced vasodilation in human hand veins. Clin Pharmacol Ther 1997; 61: $677-683$.

4 Zhang X, Hintze TH. Amlodipine releases nitric oxide from canine coronary microvessels; an unexpected mechanism of action of a calcium.channel blocking agent. Circulation 1998; 97: $576-580$.

5 Taddei S, Virdis A, Ghiadoni L, et al. Effect of calcium antagonist and beta blockade treatment on nitric oxide dependent vasodilation and oxidative stress in essential hypertensive patients. J Hypertension 2001; 19: $1379-1386$

6 Sanchez-Chapula JA, Salinas-Stefanon E, Torres-Jacomw J, Benavides DE, Navarro-Polanco RA. Blockade of currents by the antimalarial drug chloroquine in feline ventricular myocytes. J Pharmacol Exp Ther 2001; 297: 437-445.

\section{Amlodipine and grapefruit juice}

We have with interest read the paper by Vincent et al. [1] published very recently in the journal. We agree with their conclusion that grapefruit juice has no appreciable effect on amlodipine pharmacodynamics and pharmacokinetics in patients in general. We did in a paper some years ago show a pharmacokinetic interaction between amlodipine and grapefruit juice in healthy volunteers [2]. $C_{\max }$ as well as AUC were significantly $(P<0.05)$ higher when amlodipine $5 \mathrm{mg}$ was administered with grapefruit juice than with water. However, as in the paper by Vincent et al. there was no difference in BP or HR when amlodipine was taken together with grapefruit juice compared with when it was taken with water.

Vincent et al. refers to our paper and state that since we used a dose of $5 \mathrm{mg}$ our analytical method was operating near the detection limit for amlodipine concentration and that this increased the variability of our results. In our method the limit of quantification was $0.2 \mathrm{ng} \mathrm{ml}^{-1}$ and the coefficient of variation was below $15 \%$, i.e. the same as for the method used by Vincent et al. Vincent et al. do not discuss at all the interindividual variation in the pharmacokinetics due to biological factors. It has been shown in earlier studies that there is a variation in the pharmacokinetics of amlodipine including the bioavailability, which has been shown to vary between 52 and $85 \%$ in healthy volunteers [3]. Between patients the variations can be expected to be even larger. This variation is of far greater importance than the variations of the analytical methods. It also worth noting that in the paper by Vincent et al. pharmacokinetic data are presented for only half of the 20 individuals as far as oral administration is concerned. In our study none of the volunteers was excluded.

In our opinion, considering the individual variation in the pharmacokinetics of amlodipine it might be concluded that the possible interaction between grapefruit juice and amlodipine cannot be neglected in the clinical setting even though this interaction does not seem to be of great clinical significance in studies performed on healthy volunteers.

\section{Josefsson \& J. AhIner}

Department of Forensic Chemistry, National Board of Forensic Medicine, University Hospital, SE-581 85 Linköping, Sweden

Received 3 May 2001, accepted 16 November 2001

Correspondence: Professor J. Ahlner, Department of Forensic Chemistry, National Board of Forensic Medicine, University Hospital, SE-581 85 Linköping, Sweden. Fax: 00461310495

\section{References}

1 Vincent J, Harris SI, Foulds G, Dogolo LC, Willavize S, Friedman HL. Lack of effect of grapefruit juice on the pharmacokinetics and pharmacodynamics of amlodipine. Br J Clin Pharmacol 2000; 50: 455-463.

2 Josefsson M, Zackrisson A-L, Ahlner J. Effect of grapefruit juice on the pharmacokinetics of healthy volunteers in healthy volunteers. Eur J Clin Pharmacol 1996; 51: 189-193.

3 Faulkner JK, McGibney D, Chasseaud LF, Perry JL, Taylor IW. The pharmacokinetics of amlodipine in healthy volunteers after single intravenous and oral doses and after 14 repeated oral doses given once daily. $\mathrm{Br} \mathrm{J}$ Clin Pharmacol 1986; 22: 21-25. 


\section{A reply:}

In their letter to the editor, Josefsson \& Ahlner correctly admit that grapefruit juice has no appreciable effect on the pharmacokinetics and pharmacodynamics of amlodipine. However, they argue that in their paper [1], $C_{\max }$ as well as AUC were significantly $(P<0.05)$ higher when $5 \mathrm{mg}$ amlodipine was administered with $250 \mathrm{ml}$ of grapefruit juice compared with when administered with water. They claim that variability in the pharmacokinetics of amlodipine rather than variability due to analytical methods underlie the differences in the conclusions from their paper compared with the results from our study [2], although they did not indicate the confidence intervals of the ratios of their $C_{\max }$ and AUC. In addition, Josefsson \& Ahlner claim that whereas they showed data from all their subjects, we excluded one half of our subjects and implicitly skewed our results.

While pharmacokinetic variations due to biological factors are feasible, the magnitude of the mean absolute bioavailability for amlodipine reported in our study is in agreement with data reported independently by Abernethy et al. [3], as well as data on file from a study in mildly hypertensive patients. With an absolute bioavailability $>80 \%$, bioavailability enhancement as described for calcium channel blockers with a high first-pass metabolism when grapefruit juice is concurrently administered is unlikely to be significant with amlodipine, even if one assumes complete absorption. There is a correlation between blood pressure fall and plasma concentrations of calcium channel blockers [4-6], including amlodipine [3]. In studies with felodipine and nisoldipine, significant changes in exposure following concurrent administration of grapefruit juice were accompanied by changes in haemodynamics [7, 8]. The absence of any change in blood pressure or heart rate in their study is therefore consistent with the absence of any meaningful changes in blood concentrations of amlodipine.

The assertion that we excluded subjects in our analysis is a complete misrepresentation of our paper. Pharmacokinetic and pharmacodynamic data from all 20 subjects were used in the analyses of both the oral and intravenous arms of the data on unresolved amlodipine. Plasma samples collected during our study were assayed for unresolved amlodipine prior to enantiomer assays being run. In a few subjects, the volume of plasma was inadequate at all time points for the enantiomer assays. We correctly identified all such data in our manuscript. We believe that the conclusions from our study have not been compromised because of the missing enantiomer data, recognizing the consistency of the data shown.

There were also substantial differences between the conditions and design of our study and the study reported by Josefsson \& Ahlner. We used a larger sample size of
20 subjects compared with their 12 subjects. We also used a higher dose of amlodipine (10 vs $5 \mathrm{mg}$ ), to ensure a more appropriate characterization of plasma concentrations over a longer interval. In our study, grapefruit juice was administered with amlodipine on the day of dosing and repeated daily with breakfast for the 9 days during which blood samples were obtained for pharmacokinetics. This profile more clearly mimics real life situations for subjects who routinely drink grapefruit juice with breakfast when medications are commonly consumed. In addition, the conditions of our study would more readily demonstrate any potential interaction, recognizing that grapefruit juice was on board continuously. Finally, we studied both oral and intravenous amlodipine to more appropriately define its pharmacokinetics under the conditions of our study. The results with both formulations were consistent for both unresolved amlodipine and the enantiomers.

In conclusion, while it may be argued that normal biological variability could influence the pharmacokinetic data shown in these studies, it is unfortunate that Josefsson \& Ahlner misrepresented our data in their letter. We agree with the conclusion in their paper that grapefruit juice does not alter the pharmacokinetics and pharmacodynamics of amlodipine.

\section{John Vincent, Stuart Harris,' George Foulds, Lisa Dogolo, Susan Willavize \& Hylar Friedman Pfizer Global Research and Development, Groton and New London, CT and ${ }^{1}$ Sea View Research, Miami, FL, USA}

Received 26 October 2001, accepted 16 November 2001

Correspondence: Dr J. Vincent, Pfizer Global Research and Development, Groton and New London, CT, USA.

Fax: 0018604415302

\section{References}

1 Josefsson M, Zackrisson A-L, Ahlner J. Effect of grapefruit juice on the pharmacokinetics of amlodipine in healthy subjects. Eur J Clin Pharmacol 1996; 51: 189-193.

2 Vincent J, Harris SI, Foulds G, Dogolo LC, Willavize S, Friedman HL. Lack of effect of grapefruit juice on the pharmacokinetics and pharmacodynamics of amlodipine. Br J Clin Pharmacol 2000; 50: 455-463.

3 Abernethy DR, Gutkowska J, Winterbottom LM. Effects of amlodipine, a long-acting dihydropyridine calcium antagonist in aging hypertension: Pharmacodynamics in relation to disposition. Clin Pharmacol Ther 1990; 48: 76-86.

4 Kleinbloesem $\mathrm{CH}$, van Brummelen P, van de Linde JA, Voogd PJ, Breimer DD. Nifedipine kinetics and dynamics in healthy subjects. Clin Pharmacol Ther 1984; 35: 742-749.

5 Pasanisi F, Reid JL. Plasma nifedipine levels and fall in blood pressure in a 53 year old woman. Eur J Clin Pharmacol 1983; 25: 143-144. 
6 Aoki K, Sato K, Kawaguchi Y, Yamamoto M. Acute and long-term hypotensive effects and plasma concentrations of nifedipine in patients with essential hypertension. Eur J Clin Pharmacol 1982; 23: 197-201.

7 Takanaga H, Ohnishi A, Murakami H, et al. Relationship between time after intake of grapefruit juice and the effect on pharmacokinetics and pharmacodynamics of nisoldipine in healthy subjects. Clin Pharmacol Ther 2000; 67: 201-214.

8 Bailey DG, Malcolm J, Arnold O, Spence JD. Grapefruit juice-drug interactions. Br J Clin Pharmacol 1998; 46: 101-110.

\section{$\beta$-adrenoceptor blockers and serotonin}

I read with much interest the article by Sørensen et al. [1], which investigated the risk of suicide in users of antihypertensive drugs, and noted several flaws that I would like to comment on, as follows.

First, Sørensen et al. [1] concluded that users of medium and high lipid soluble $\beta$-adrenoceptor blockers may have an increased risk of suicide. However, there was no significant increase in the standardized mortality ratio (SMR) relative to suicide in users of $\beta$-adrenoceptor blockers with medium lipid solubility; note that the range of the $95 \%$ confidence interval $(0.8,2.8)$ contained 1. Therefore, their findings do not suggest that $\beta$-adrenoceptor blockers with medium lipid solubility increase suicide risk.

Secondly, there are pharmacologically heterogeneous subtypes other than lipid solubility among $\beta$-adrenoceptor blockers, although Sørensen et al. simply state that the lipid soluble $\beta$-adrenoceptor blockers pass the blood brain barrier and that biogenic amine depletion may play a role in the relation between antihypertensive medication and depression. However, 'biogenic amine depletion' is not accurate, and several $\beta$-adrenoceptor blockers, such as pindolol, have antagonistic activity at somatodendritic $5-\mathrm{HT}_{1 \mathrm{~A}}$ autoreceptors; dampen its negative feedback; and thereby increase 5-HT release. In point of fact, several studies have demonstrated that pindolol combined with selective serotonin reuptake inhibitors would result in rapid and augmented antidepressant effects [2-4]. On the other hand, metoprolol does not have such an effect at somatodendritic 5- $\mathrm{HT}_{1 \mathrm{~A}}$ autoreceptors [4]. Recently, Rösönen et al. [5] showed that the use of pindolol per se was associated with a slightly but significantly lower rate of antidepressant use when compared with that of other $\beta$-adrenoceptor blockers, which indicates that pindolol alone may have some beneficial effects on mood. Therefore, pindolol might have been found to decrease suicide if SMR in pindolol users had been calculated separately.

In conclusion, the classification of $\beta$-adrenoceptor blockers according to degree of lipid solubility is not sufficient relative to discussion of depression and/or suicide, and antagonistic activity at somatodendritic 5- $\mathrm{HT}_{1 \mathrm{~A}}$ autoreceptors should be taken into consideration. At the very least, pindolol and metoprolol should not be categorized in the same group.

\section{Takeshi Terao}

Department of Psychiatry, University of Occupational and Environmental Health School of Medicine, Kitakyushu, Japan

Received 6 November 2001, accepted 13 December 2001.

Correspondence: Takeshi Terao, MD, PhD, Associate Professor, Department of Psychiatry, University of Occupational and Environmental Health School of Medicine, Yahatanishi-ku, Kitakyushu 807-8555, Japan. Tel: +81-93-691-7253;

Fax: +81-93-692-4894; E-mail: t-terao@med.uoeh-u.ac.jp

\section{References}

1 Sørensen HT, Mellemkjær L, Olsen JH. Risk of suicide in users of $\beta$-adrenoceptor blockers, calcium channel blockers and angiotensin converting enzyme inhibitors. Br J Clin Pharmacol 2001; 52: 313-318.

2 Pérez V, Gilaberte I, Faries D, Alvarez E, Artigas F. Randomised, double-blind, placebo-controlled trial of pindolol in combination with fluoxetine antidepressant treatment. Lancet 1997; 349: 1594-1597.

3 Bodet R, Thomas P, Dupuis B. Effect of pindolol on onset of action of paroxetine in the treatment of major depression: intermediate analysis of a double-blind, placebo-controlled trial. Am J Psychiatry 1998; 155: 1346-1351.

4 Zanardi R, Artigas F, Franchini L, et al. How long should pindolol be associated with paroxetine to improve the antidepressant response? J Clin Psychopharmacol 1997; 17: $446-450$.

5 Rösönen P, Hakko H, Tihonen J. Pindolol and major affective disorders: a three-year follow-up study of 30,485 patients. J Clin Psychopharmacol 1999; 19: 297-302.

\section{Confidence intervals, misclassification of exposure and risk of suicide in users of $\beta$-adrenoceptor blockers: a reply}

Thank you very much for giving us the opportunity to comment on the letter of Professor Terao.

Our study is an observational follow-up study, where judgement of causality should not only be based on whether the confidence interval includes the value 1 . In our opinion judgement of causality should be based on the risk estimate and confidence interval with careful examination of possible bias and confounding. Only when the exposure is randomized, do we have the solid 
theoretical basis for probability models from which $P$ values are derived [1].

We agree that the classification of $\beta$-adrenoceptor blockers may be too simple from a pharmacological point of view [2]. If there is any difference in risk of suicide between subjects included in the medium lipid solubility group of $\beta$-adrenoceptor blockers, and in particular if some of the $\beta$-adrenoceptor blockers included in this group are not associated with suicide, this misclassification simply leads to an underestimation of the real risk estimate.

\section{Henrik Toft Sørensen,' Lene Mellemkjær ${ }^{2}$ \& Jørgen H. Olsen ${ }^{2}$}

${ }^{1}$ Department of Clinical Epidemiology, Aarhus University Hospital and Aalborg Hospital, Vennelyst Boulevard 6, Building 260, 8000 Århus C and ${ }^{2}$ The Danish Cancer Society, Institute of Cancer Epidemiology, Strandboulevarden 49, 2100 Copenhagen Ø, Denmark

Received 19 November 2001, accepted 17 December 2001

Correspondence: Dr H. T. Sorensen, Department of Clinical Epidemiology, Aarhus University Hospital and Aalborg Hospital, Vennelyst Boulevard 6, Building 260, 8000 Århus C, Denmark

\section{References}

1 Poole C. Low P-values or narrow confidence intervals: which are more durable? Epidemiology 2001; 12: 291-297.

2 Svendsen TL. Beta-receptorblokkerende midler. In Lagemiddelkataloget. Copenhagen: Dansk Lagemiddel Information eds Kristensen MB, Friis $\mathrm{H}$, Rassing MR. 1998: 179-183.

\section{CYP2C9 polymorphism and warfarin dose requirements}

We were interested to read Dr Goldstein's review on the clinical relevance of genetic polymorphisms in the human CYP2C subfamily [1]. While we believe this to be a generally wide-ranging and useful contribution, we were surprised to read that a recent paper by us reporting that CYP2C9 genotype was a good predictor of warfarin dose requirement [2] was said to involve the use of a PCR assay with primers which were not specific for CYP2C9 but also amplified CYP2C19. The review therefore suggests that our study might have underrepresented the incidence of the 'PM allele' in the low warfarin dose requirement group. Though we agree that the study of Yasar and colleagues [3] indicated that the primers we used for CYP2C9 genotyping, which were originally designed by Wang et al. [4], might amplify other related sequences at a low level, we were aware of this confounder prior to the commencement of our warfarin study. We found that the problem could be avoided by using the maximum annealing temperatures $\left(60^{\circ} \mathrm{C}\right.$ for CYP2C $9 \star 2$ and $61^{\circ} \mathrm{C}$ for CYP2C9 3 for $2 \mathrm{~min}$ on a Techne PHC-3 heating block), compatible with successful PCR amplification. Restriction digests of samples homozygous for

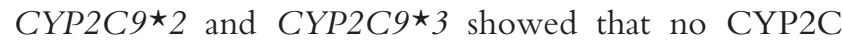
sequences other than CYP2C9 were amplified.

We believe that the accuracy of the assay in our hands is also confirmed by the fact that in our control group, we obtained similar genotype distributions to those obtained by others for Northern European populations (see Table 1). In addition, our findings with respect to warfarin dose requirement and CYP2C9 genotype have been replicated in a recent large study [5] which used different PCR primers for CYP2C9 amplification. While the finding that $C Y P 2 C 9 \star 2$ heterozygotes have a lower warfarin dose requirement to homozygous wild-type subjects might seem unexpected in the light of early studies using a yeast expression system which suggested that the Arg144Cys substitution did not affect S-warfarin 7-hydroxylation [6], others have observed a highly significant decrease in $V_{\max }$ for $S$-warfarin 7-hydroxylation by this substitution in a human cell line expression system [7]. This discrepancy between expression systems may be due to differences in cytochrome P450-cytochrome P450 oxidoreductase ratios between human and yeast cell lines [8]. S-warfarin does not appear to be the only CYP2C9 substrate where the CYP2C9 2 allele gene product shows functionally significant differences in catalytic activity from the wild-type since individuals heterozygous for CYP2C9 2 have also been demonstrated to show slower metabolism of phenytoin compared with homozygous wild-type individuals [9].

The findings that individuals heterozygous for

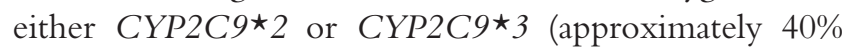
of Northern Europeans) as well as those with two variant CYP2C9 alleles (approximately 3\% of Northern Europeans) have on average a lower warfarin dose requirement than homozygous wild-type individuals are,

Table 1 CYP2C9 genotype frequencies in Northern European controls.

\begin{tabular}{lccc}
\hline & $\begin{array}{c}\text { UK [2] } \\
\mathrm{n}=100\end{array}$ & $\begin{array}{c}\text { UK [10] } \\
\mathrm{n}=100\end{array}$ & $\begin{array}{c}\text { Sweden [3] } \\
\mathrm{n}=430\end{array}$ \\
\hline$\star 1 / \star 1$ & $60(0.60)$ & $62(0.62)$ & $287(0.67)$ \\
$\star 1 / \star 2$ & $20(0.20)$ & $19(0.19)$ & $80(0.19)$ \\
$\star 1 / \star 3$ & $17(0.17)$ & $15(0.15)$ & $50(0.12)$ \\
$\star 2 / \star 3$ & $2(0.02)$ & 0 & $8(0.02)$ \\
$\star 2 / \star 2$ & 0 & $3(0.03)$ & $2(0.005)$ \\
$\star 3 / \star 3$ & $1(0.01)$ & $1(0.01)$ & $3(0.01)$ \\
\hline
\end{tabular}


we believe, of particular clinical relevance in view of the large number of patients who fall into these categories.

\section{Ann K. Daly, ${ }^{1,2}$ Christopher P. Day² \& Guruprasad P. Aithal ${ }^{3}$ \\ ${ }^{1}$ Department of Pharmacological Sciences and ${ }^{2}$ Centre for Liver Research, University of Newcastle Medical School, Framlington Place, Newcastle upon Tyne NE2 $4 \mathrm{HH}$ and ${ }^{3}$ Queen's Medical Centre University Hospital, $D$ floor, South block, Nottingham NG7 $2 U H$}

Received 23 November 2001, accepted 10 December 2001

Correspondence: Dr A. K. Daly, Department of Pharmacological Sciences University of Newcastle Medical School, Framlington Place, Newcastle upon Tyne NE2 4HH, UK. Tel.: 0191 2227031; Fax: 0191 2227230; E-mail: A.K.Daly@ncl.ac.uk

\section{References}

1 Goldstein JA. Clinical relevance of genetic polymorphisms in the human CYP2C subfamily. Br J Clin Pharmacol 2001; 52: 349-355.

2 Aithal GP, Day CP, Kesteven PJL, Daly AK. Association of polymorphisms in the cytochrome $\mathrm{P} 450 \mathrm{CYP} 2 \mathrm{C} 9$ with warfarin dose requirement and risk of bleeding complications. Lancet 1999; 353: 717-719.

3 Yasar U, Eliasson E, Dahl ML, Johansson I, Ingelman-Sundberg M, Sjoqvist F. Validation of methods for CYP2C9 genotyping: Frequencies of mutant alleles in a Swedish population. Biochem Biophys Res Comm 1999; 254: 628-631.

4 Wang S-L, Huang J-D, Lai M-D, Tsai J-J. Detection of CYP2C9 polymorphism based on the polymerase chain reaction in Chinese. Pharmacogenetics 1995; 5: 37-42.

5 Taube J, Halsall D, Baglin T. Influence of cytochrome P-450CYP2C9 polymorphisms on warfarin sensitivity and risk of over-anticoagulation in patients on long-term treatment. Blood 2000; 96: 1816-1819.

6 Kaminsky LS, Demorais SMF, Faletto MB, Dunbar DA, Goldstein JA. Correlation of human cytochrome P4502c substrate specificities with primary structure - warfarin as a probe. Mol Pharmacol 1993; 43: 234-239.

7 Rettie AE, Wienkers LC, Gonzalez FJ, Trager WF, Korzekwa KR. Impaired (S)-warfarin metabolism catalysed by the R144C allelic variant of CYP2C9. Pharmacogenetics 1994; 4: 39-42.

8 Crespi CL, Miller VP. The R144C change in the CYP2C9 2 allele alters interaction of the cytochrome P450 with NADPH. cytochrome P450 oxidoreductase. Pharmacogenetics 1997; 7: 203-210.

9 Aynacioglu AS, Brockmoller J, Bauer S, et al. Frequency of cytochrome P450CYP2C9 variants in a Turkish population and functional relevance for phenytoin. Br J Clin Pharmacol 1999; 48: 409-415

10 Stubbins MJ, Harries LW, Smith G, Tarbit MH, Wolf CR. Genetic analysis of the human cytochrome P450 CYP2C9 locus. Pharmacogenetics 1996; 6: 429-439.

\section{CYP2C9 polymorphisms and CYP2C9*2 genotyping primers}

The primers first suggested by Wang et al. [1] for the genotyping of the CYP2C9 Arg144Cys mutation were based on the gene structure of CYP2C9 prior to knowledge of the gene structure of the closely related CYP2C19. Early studies cannot be faulted for using these primers, since the gene structure of CYP2C19 was not known. The primers utilized by Wang et al. [1] differ from the gene structure of CYP2C19 by only a few bases (underlined) [5'-GAATATGAAGCAGTGAAGGAA3-3' (forward primer) and 5'-GGECTTGGTTTTTCTCAACTC-3' (reverse primer)]. In particular, mismatches at the $5^{\prime}$-end are not generally considered to confer much specificity to primers, since many laboratories add restriction sites in this way. The primers later described by Sullivan-Klose et al. [2, 5'-TACAATACAATGAAAATACATG-3' and 5'-CTAACĀACCAGACTCAT $\left.\overline{A A T G}-3^{\prime}\right]$ included a number of mismatches with $\mathrm{CYP} 2 \mathrm{C} 19$ (underlined) including two at the 3 '-end of each primer. Thus, I have always considered them to be more specific for CYP2C9 and to be less likely to amplify the closely related CYP2C19. Since both CYP2C9 and CYP2C19 contain an arginine at 144, any spurious amplification of CYP2C19 would make it more difficult to recognize homozygous CYP2C9 2 individuals. However any comments of mine concerning the use of the primers described by Wang [1] by any subsequent particular group were unfortunate and not very tactful. Many groups have used these primers. Dr Daly's group has had extensive experience with genotyping tests, and she states that the annealing temperatures she used allowed her to avoid the coamplification problems. Her laboratory in fact identified three homozygous $C Y P 2 C 9 \star 2$ individuals. Other laboratories including mine and those of Yasar et al. [3] have had problems with specificity using the primers described by Wang et al. [1]. The study of Yasar et al. [3] (Figure 1, panel 1) indicates heavy contamination by non-CYP2C9 genes, not slight contamination as suggested by Dr Daly. AvaII should not digest the PCR products of an individual homozygous for the CYP2C9 2 allele, but when Yasar et al. [3] used the primers described by Wang, approximately $50 \%$ of the PCR products appeared to be cleaved. Such results would have led to the incorrect identification of the individual as heterozygous for CYP2C $9 \star 2$. Yasar et al. [3] were not able to achieve specificity using these primers by changing either the annealing temperature or $\mathrm{Mg}^{2+}$. I would simply like to point out that the choice of alternate primers containing more base differences with CYP2C19 would seem to be a prudent precaution in the genotyping of $C Y P 2 C 9 \star 2$ in future studies. The use of more specific primers may help many laboratories to achieve the specificity 
necessary for the identification and study of homozygous CYP2C9 2 individuals. The identification and clinical testing of metabolism of drugs by individuals homozygous for the CYP2C9 2 alleles is an important issue. Compared with $C Y P 2 C 9 \star 3$, there is a paucity of studies in the literature concerning rates of metabolism of drugs in CYP2C9 2 homozygous individuals.

I hope my review indicated that I agree with Dr Daly that CYP2C9 2 is indeed a defective allele with lower clearances for many drugs than the wild-type CYP2C9. Although our early cDNA expression studies did not detect differences between the catalytic activity of the CYP2C9 2 allele and the wild-type allele in a yeast cDNA expression, this may have been due to the cDNA expression system used. Of the CYP2C9 alleles reported to date, CYP2C9 3 , $C Y P 2 C 9 \star 4$, and CYP2C9*5 have dramatically impaired catalytic activity [4]. The CYP2C9*4 [4] (Ile359Thr) and

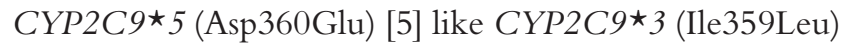
alleles all contain changes at amino acids 359 and 360 which are predicted to lie in a putative substrate binding site. These alter either the $K_{m}$ or $V_{\max }$ toward various substrates. A new null allele of CYP2C9 has been discovered with even more deleterious effects in vivo [6]. However, numerous studies have verified that the CYP2C9*2 protein has moderately impaired catalytic activity in vitro perhaps due to impaired binding of the reductase [7]. There are also studies of heterozygous individuals showing some impairment in metabolism of certain substrates in vivo. This is important because of (a) the relatively high frequency of the CYP2C9*2 allele (approximately 11\%), the high frequency of heterozygotes, and (b) the fact that CYP2C9 mediates metabolism of several clinically important drugs with narrow therapeutic indices such as phenytoin and warfarin.

\section{Joyce A. Goldstein}

National Institute of Environmental Health Services, PO Box 12233, Research Triangle Park, NC 27709, USA

Received 4 December 2001, accepted 17 December 2001

Correspondence: Dr J. A. Goldstein, National Institute of Environmental Health Services, PO Box 12233, Research Triangle Park, NC 27709, USA. E-mail: goldste1@niehs.nih.gov

\section{References}

1 Wang SL, Huang J, Lai MD, et al. Detection of CYP2C9 polymorphism based on the polymerase chain reaction in Chinese. Pharmacogenetics 1995; 5: 37-42.

2 Sullivan-Klose TH, Ghanayem BI, Bell DA, et al. The role of the CYP2C9-Leu359 allelic variant in the tolbutamide polymorphism. Pharmacogenetics 1996; 6: 341-349.

3 Yasar U, Eliasson E, Dahl ML, et al. Validation of methods for CYP2C9 genotyping: frequencies of mutant alleles in a Swedish population. Biochem Biophys Res Commun 1999; 254: 628-631.

4 Ieiri IMK, Urae A, Wada Y, et al. Stereoselective 4'-hydroxylation of phenytoin. Relationship to (S)-mephenytoin polymorphism in Japanese. Br J Clin Pharmacol 1997; 43: 441-445.

5 Dickmann LJ, Rettie AE, Kneller MB, et al. Identification and functional characterization of a new CYP2C9 variant (CYP2C9 5 ) expressed among African Americans. Mol Pharmacol 2001; 60: 382-387.

6 Kidd RS, Currey TB, Gallagher S, Edeki T, Blaisdell J, Goldstein JA. Identification of a null allele of CYP2C9 in an African-American exhibiting toxicity to phenytoin. Pharmacogenetics 2002; 11: 803-815.

7 Crespi CL, Miller VP. The R144C change in the CYP2C9 2 allele alters interaction of the cytochrome $\mathrm{P} 450$ with NADPH. cytochrome P450 oxidoreductase. Pharmacogenetics 1997; 7: 203-210. 